\title{
RESUMEN
}

Durante las últimas décadas, las grandes empresas han tenido que adaptarse a los desafíos actuales, los cuales requieren de un mayor compromiso ambiental y con la sociedad, según autores los enfoques de estrategias han pasado de ser únicamente económicos, enfocados también en el medio ambiente y la sociedad, así generar un compromiso de sostenibilidad con los distintos grupos de interés.

El propósito de la investigación es analizar la implementación de los sistemas de gestión ambiental en siete empresas mineras de Chile, que fueron seleccionadas en base a sus niveles de producción y contribución para el país, con el propósito de aportar al proceso de aplicación de estos sistemas, en dichas organizaciones.

La metodología se lleva a cabo con enfoque cualitativo y un alcance de sintetización. Para la obtención de información, la investigación está basada en diversas fuentes, tales como, memorias anuales y reportes de sustentabilidad de los sujetos de investigación, además se utilizan artículos de revistas, páginas web oficiales, informes de organismos públicos nacionales y bases de datos. Con ayuda del software de análisis cualitativo: ATLAS.ti se alcanzaron los objetivos propuestos. Como resultado, la implementación de los SGA es fundamental para la continuidad de la industria, establecer la sustentabilidad como un valor de base para las operaciones, permite integrar la eficiencia energética y fortalecer sus sistemas de control y gestión. También se observa una falta de transparencia en cuanto a revelación de cifras en torno mejoramiento ambiental y financiación de estos, provocando cuestionamientos para el usuario.

Palabras clave: empresas mineras, impacto ambiental, sistemas de gestión ambiental sustentabilidad.

\section{ANÁLISIS DE LA IMPLEMENTACIÓN DE UN SISTEMA DE GESTIÓN AMBIENTAL EN EMPRESAS MINERAS DE CHILE.}

Antonia Carvajal Ahumada - Francisca González González 2 - Iván Cubillos Garrido ${ }_{3}$

\section{Fechas de recepción: 15 de Octubre de 2021}

Fecha de aceptación: 12 de Diciembre de 2021

\section{DOI: http://doi.org/10.22370/riace.2021.10.1.3089}

1 Licenciada en Sistemas de Información Financiera y Control de Gestión, Escuela de Auditoría. Universidad de Valparaíso.

2 Licenciada en Sistemas de Información Financiera y Control de Gestión, Escuela de Auditoría. Universidad de Valparaíso.

3. Escuela de Auditoría. Universidad de Valparaíso. 


\title{
ANALYSIS OF THE IMPLEMENTATION OF AN ENVIRONMENTAL MANAGEMENT SYSTEM IN MINING COMPANIES IN CHILE
}

\begin{abstract}
In recent decades, large companies have had to adapt to current challenges, which require greater commitment to the environment and society. According to authors, strategy approaches have shifted from being solely economic, also focusing on the environment and society, thus generating a commitment to sustainability. with the different interest groups.

The purpose of this research is to analyze the implementation of environmental management systems in seven mining companies in Chile, which were selected based on their levels of production and contribution to the country, with the purpose of contributing to the process of applying these systems, in these organizations.

The methodology is carried out with a qualitative approach and a scope of synthetization. In order to obtain information, the research is based on various sources, such as annual reports and sustainability reports of research subjects, in addition, articles from journals, official websites, reports of national public bodies and databases are used. With the help of qualitative analysis software: Atlas.ti achieved the proposed objectives.

As a result, the implementation of the EMS is critical to the continuity of the industry, establishing sustainability as a base value for operations, enabling energy efficiency to be integrated and its control and management systems strengthened. There is also a lack of transparency in terms of the disclosure of figures on environmental improvement and financing of these, causing questions for the user.
\end{abstract}

Keywords: mining companies, environmental impact, environmental management systems sustainability. 


\section{INTRODUCCIÓN}

Debido a las crisis socioambientales, hoy en día la protección del medio ambiente ha tomado relevancia, presionando a la sociedad a tomar conciencia y acción concreta frente a dicha temática. Informes de la Organización de las Naciones Unidas (2019) declaran que el cambio climático es irrefutable, pero también indica que no es demasiado tarde para detener su avance. Lo anterior, implica que harán falta transformaciones fundamentales en todos los aspectos de la sociedad, principalmente en crear conciencia social en este aspecto.

A raíz de las declaraciones que han surgido durante los últimos años, las nuevas generaciones han desarrollado conciencia al respecto, preservar los recursos naturales y encontrar soluciones sostenibles es su objetivo. En este contexto, de acuerdo con Alaña et al. (2017) nacen nuevas tendencias de administración, que van en línea con el cumplimiento de una responsabilidad social, aquellas favorecen a la aplicación de regulaciones, procedimientos y modelos que se ajustan a incrementar la sostenibilidad y eficiencia de los procesos. No obstante, para que se pueda llevar a cabo, es necesario que los directivos y gobierno corporativo de las entidades utilicen sus habilidades, demostrando su liderazgo por medio de la innovación de sus metodologías, dando prioridad a la responsabilidad social ambiental y de esta manera estimular a los trabajadores, logrando que se identifiquen con los nuevos objetivos y metas definidas.

Las empresas tienen carácter de sistema, el entorno es uno de los factores a considerar, por tanto, lo que ocurra en él afectará internamente a la entidad. La instauración y autorregulación de cambios en materias de gestión ambiental en un sistema tal como una organización, implica que esta realice un esfuerzo importante para dar paso a nuevas políticas. Según Coglianese \& Nash (2001) adaptarse a nuevas maneras de ejecutar los procesos implica que la fuerza laboral tenga la disposición y compromiso con estos, de lo contrario, se convierte en una adversidad que afecta significativamente a la cultura organizacional. En cuanto a la definición y aplicación de diferentes fórmulas que favorezcan al rendimiento ambiental, como lo menciona Sumba \& Lourdes (2018), es primordial que desde la base de su proceso administrativo, estén claramente definidos los objetivos específicos, misión y visión pues entorno a ellos, se enfocarán las actividades a realizar por los colaboradores.

La Globalización ha sido definida por Giddens (1999) como un proceso complejo, compuesto por múltiples partes que se interrelacionan y se ven afectadas por la dependencia e interdependencia de factores geográficos, políticos, culturales y económicos, entre otros. Esto quiere decir, que hay acontecimientos que no se pueden controlar y que simplemente el ser humano se adapta a esto, incluso hasta el punto de ni siquiera percibir cómo se han estructurado los modos de vivir, profundamente a lo largo del tiempo.

Este complejo proceso genera una evolución en un periodo de tiempo, marcando 
una época de transformaciones de muchos ámbitos, entonces "se considerará al proceso de globalización como un proceso evolutivo económico, político y social que se desarrolla en el período histórico de la globalización" Palomo (2012, p.1). Dichas transformaciones no están exentas de consecuencias y sobre todo con el acelerado crecimiento industrial y tecnológico del que hoy en día se es testigo.

Es complejo a estas alturas y bajo este sistema alcanzar una vida totalmente sostenible, en estos momentos el planeta se encuentra en un estado de deuda ecológica. El daño y las crisis son inminentes, afectarán a las futuras generaciones y por eso y más, es labor de todos los países hallar la forma de hacer lo que sea necesario para frenar lo máximo posible la devastación del planeta tierra.

Según Kammerbauer (2001) el desarrollo de una sociedad se manifiesta como un proceso con un conjunto de oportunidades que incrementan el bienestar para los individuos y la colectividad. Para que el crecimiento pueda continuar satisfaciendo las necesidades humanas es primordial que se pueda tener una visión donde el medio ambiente y desarrollo trabajen en forma conjunta teniendo en consideración las consecuencias de los impactos ambientales negativos.

Para efectos de la investigación se define el término "sostenible", como aquel "que proviene de una raíz económica y se basa en la posibilidad de que cualquier sistema sea capaz de sostenerse sin tener que recurrir a un mayor consumo de energía." (Perea et al. 2009, p. 7)

La Asamblea General de las Naciones Unidas en su informe Brundtland, con el objetivo de generar conciencia para preservar el medio ambiente, logra definir el desarrollo sostenible como "el desarrollo que satisface las necesidades del presente sin comprometer la capacidad de las generaciones futuras de satisfacer las propias". Gallopín (1987, p. 23).

A lo largo de la historia, muchas civilizaciones antiguas han sufrido las consecuencias de agotar sus recursos y simplemente han colapsado, demostrando que "las sociedades no sostenibles se derrumban" Mulder (2007, p.13), esto se ha debido al uso de métodos no sustentables de agricultura, por ejemplo, existen evidencias que señalan que la irrigación en esta actividad, contribuyó al derrumbe de las civilizaciones de los Mayas en América Central, del valle Indo en el sur de Asia y de las ciudades de Mesopotamia en el Oriente Medio. También está el caso de la erosión la cual en la década de los 30’ originó un grave problema en el medio oeste de los Estados Unidos y ha destruido comunidades en todas las partes del mundo.

Como lo describe Pérez (2019) la Asamblea General de la ONU, el 25 de septiembre de 2015, adoptó la Agenda 2030 para el Desarrollo Sostenible. Los objetivos, conocidos ya mundialmente como los Objetivos para el Desarrollo Sostenible (ODS) han sido recogidos en un documento titulado «Transformar nuestro mundo: la Agenda 2030 para el Desarrollo Sostenible» estos objetivos configuran el marco a través del cual los países podrán dirigir sus esfuerzos a luchar contra el cambio 
climático, poner fin a la pobreza en todas sus formas, reducir la desigualdad, trazar un plan de acción a favor de las personas, el planeta y la prosperidad, que también tiene la intención de fortalecer la paz universal y el acceso a la justicia.

Actualmente, se está inmerso en un profundo proceso de cambios. Con la aparición del concepto de "desarrollo sostenible" ya existe una aceptación prácticamente universal respecto a ello. Según lo que mencionan los autores Acuña et al. (2017) es importante considerar que el desarrollo sostenible se basa en la visión "triple bottom", que integra los factores económicos, sociales y ambientales en las actividades productivas, influenciando en las estrategias de las organizaciones.

Los comportamientos competitivos de las empresas en la actualidad, la multiculturalidad, la calidad, entre otros factores socioeconómicos dan nacimiento al concepto de gestión en una administración moderna.

Para el cumplimiento de los objetivos de toda organización, es necesario tener la habilidad para poder diseñar e implementar las estrategias, dicha habilidad se puede definir como administración o gestión la cual "consiste en coordinar las actividades de trabajo de modo que se realicen de manera eficiente y eficaz con otras personas y a través de ellas." Robbins \& Coulter (2005, p. 7). Otros autores definen la gestión como "un proceso que comprende determinadas funciones y actividades organizativas que los gestores deben llevar a cabo con el fin de lograr los objetivos y metas deseadas." Muriel (2006, p. 2).

En base a estos conceptos Van Hoof et al. (2008) logran definir el objetivo de la gestión ambiental, el cual busca el equilibrio entre la contaminación ambiental y los procesos de desarrollo económico, además de integrar el manejo de la variable ambiental como un pilar fundamental para su competitividad y desarrollo sostenible. En una organización el sistema de gestión ambiental proporcionará un marco para que la empresa pueda tomar decisiones hacia un rumbo más eficiente.

La definición de sistema de gestión ambiental para efecto de esta investigación se establece como la parte del sistema general de gestión de una empresa, que incluye: la estructura organizacional, planificación de las actividades, responsabilidades prácticas, procedimientos, procesos y recursos para desarrollar, implementar, revisar y mantener dicho sistema. International Organization for Standardization (2015).

No obstante, bajo la definición de otros autores, la gestión ambiental es "un proceso de toma de decisiones relacionadas con el manejo de la variable ambiental en el diseño e implementación de sistemas de gestión en el interior de las empresas y otras organizaciones." Van Hoof et al. (2008, p. 45).

A partir de otro enfoque, la gestión ambiental es "un mecanismo formal y estructurado que proporciona un marco de acción riguroso necesario para alcanzar y mantener un desempeño ambiental eficiente y efectivo". Moreno \& Rosero (2000, p. 52) Esto propone que la gestión ambiental va más allá de cumplir 
con una responsabilidad, sino que se trata de una disciplina estructurada que las organizaciones deberán cumplir.

Desde el punto de vista de Prieto (2011) la implementación de una gestión ambiental puede brindar la oportunidad a las organizaciones de que puedan obtener beneficios económicos que se logren reflejar en sus resultados financieros, por medio del cuidado y protección del medio ambiente en el que ejercen sus operaciones. Para ello será muy importante que la dirección y líderes de la empresa, se encuentren especialmente activos al implementar estas decisiones, motivando y comprobando que los objetivos se sigan en cada área.

Según el mismo autor, la implantación de un sistema de gestión ambiental en conformidad con las normas ISO, proporciona un carácter certificable a la empresa, lo que le permite ser reconocida en base al criterio de sistemática mejora continua "PHVA" es decir, planificar, hacer, verificar y actuar, proceso que acompañan la visión de mejorar el comportamiento ambiental de las empresas en base a sus objetivos y mejoras en la gestión, esto puede otorgar variadas ventajas como por ejemplo el reconocimiento de terceros y una presencia que refleja liderazgo y confianza.

Reafirmando lo anterior en base a la misma normativa ISO 14.001 (2015), el modelo PHVA proporciona un proceso iterativo usado por las organizaciones para lograr la mejora continua, el cual se puede aplicar a un sistema de gestión ambiental y a cada uno de sus elementos individuales, y se puede describir brevemente así:

A) Planificar: establecer los objetivos ambientales y los procesos necesarios para generar y proporcionar resultados de acuerdo con la política ambiental de la organización.

B) Hacer: implementar los procesos según lo planificado.

C) Verificar: hacer el seguimiento y medir los procesos respecto a la política ambiental, incluidos sus compromisos, objetivos ambientales y criterios operacionales, e informar de sus resultados.

D) Actuar: emprender acciones para mejorar continuamente.

El desafío de hoy para las organizaciones radica en satisfacer a sus clientes por medio del desempeño excepcional de los procesos. Uno de los procesos fundamentales que se deben tener en cuenta al analizar el desempeño de una organización es la mejora continua. Los autores han definido el enfoque de la mejora continua, "comprende tanto una filosofía como un sistema destinado a mejorar en materia: de niveles de calidad, productividad, costos, niveles de satisfacción, niveles de seguridad, tiempos totales de los diversos ciclos, tiempos de respuesta y grado de fiabilidad de los procesos." Esquivel et al. (2017, p. 59). Sin duda será importante la colaboración de los empleados para mantener el proceso de mejora continua, acompañado de un buen liderazgo y dirección que facilite la adaptación de la cultura organizacional a este sistema. 
Como lo destaca Becerra et al. (2019) el mejoramiento continuo es uno de los principios fundamentales de la calidad, es por ello por lo que Shewhart diseña la base de los sistemas de calidad actuales, el ciclo PHVA (Planear, Hacer, Verificar, Actuar), bautizado más adelante por los japoneses como el ciclo Deming.

Para el propósito de esta investigación, se debe tomar en cuenta que "la mejora del desempeño se relaciona con el uso de un SGA para mejorar el desempeño ambiental en coherencia con la política ambiental de la organización." International Organization for Standardization. (2015).

La gestión ambiental debe lograr ser parte del sistema general de gestión de cualquier organización, razón por la que se debe definir los objetivos para lograr los resultados que desea. Como consecuencia, Acuña et al. (2017) establecen los principales objetivos de la gestión ambiental:

1.- Identificar los aspectos ambientales significativos, al igual que la legislación aplicable y otros requisitos.

2.- Formular y asumir el compromiso de política ambiental.

3.- Concretar el compromiso con objetivos, metas y acciones específicas;

4.- Lograr los objetivos y metas previstas.

A pesar de lo anterior, no se puede dejar de lado el alcance de la ISO 14.001, debido a que esta normativa, otorga el modelo a seguir para poder implantar un sistema de gestión ambiental en la empresa, el propósito de este instrumento es dar un marco de referencia para la protección del medio ambiente

Las organizaciones en la actualidad se encuentran en la búsqueda de acciones para generar un desarrollo sostenible. La subsistencia de las organizaciones depende de la interacción con el entorno para lograr adaptarse y obtener de ello ventajas competitivas en un libre mercado. Se hace necesario que las empresas puedan identificar las estrategias competitivas para hacer frente a las exigencias de los mercados, definiendo a la estrategia como el "plan de acción que sigue la administración para competir con éxito y obtener utilidades, a partir de un arsenal integrado de opciones. La formulación de la estrategia representa el compromiso de la administración de emprender un conjunto particular de acciones." Thompson et al. (2012, p.4).

En base a ello existen tres estrategias genéricas para dar frente a los contrincantes de la industria, aquellas son definidas por Porter (1982):

1.- Liderazgo global en costos.

2.- Enfoque de diferenciación y;

3.- Enfoque o concentración. 
El entorno competitivo brinda una oportunidad a las empresas, las cuales pueden convertir el cuidado del medio ambiente en una estrategia de diferenciación la cual "diferencia el producto o servicio que ofrecemos, creando así algo que en la industria entera se percibe como único" Porter (1982, p.54).

La gestión ambiental juega un papel relevante en la diferenciación, puesto que encamina los procesos y recursos manteniendo el compromiso de la protección ambiental, eso es lo que los nuevos consumidores prefieren, marcas de productos o servicios que se encaminan a las nuevas tendencias productivas y que estén dispuestas a realizar esfuerzos por proteger el medio ambiente. Al lograr tener una forma de hacer las cosas que no sea imitable, las empresas pueden crear barreras de entrada por medio de la eco-innovación y sostenibilidad.

Según autores como Ferrari (2012) la gestión ambiental se puede considerar una ventaja competitiva a largo plazo, permitiendo a las organizaciones incrementar la eficiencia y la competitividad en el mercado, debido a que las posibilidades de diferenciación aparecen a través de elementos intangibles como en la protección del medio ambiente.

En base a este enfoque Ios SGA fueron "creados como rutas para identificar y manejar sistemáticamente los aspectos e impactos ambientales generados por parte de las empresas" Acuña et al. (2017, p.145). Con esta mirada, el objetivo para el cual la empresa implementa la estrategia de la gestión ambiental sería alcanzar la eficiencia y conseguir la ecoeficiencia. ¿Qué es la ecoeficiencia?... básicamente se refiere a producir más con menos:

Significa ofrecer la misma o mayor satisfacción al cliente con menos utilización de materia y de energía con menos contaminación y con menor producción de residuos. Significa añadir más valor a los productos y servicios. La ecoeficiencia tiene también repercusiones en el modelo de consumo. La ecoeficiencia significa superar el modelo actual de «usar y tirar» y sustituirlo por un modelo de consumo que conserve, valorice y reutilice los productos y servicios. Esta concepción obliga a la empresa a diseñar de una forma nueva sus productos y servicios y a velar activamente para que su utilización por el consumidor sea igualmente ambientalmente positiva. Ludevid (2004, p.44)

Estos conceptos "verdes" tales como desarrollo sostenible 'incluyen la ecoeficiencia como clave para armonizar el desarrollo económico con la sostenibilidad ambiental. Filippone et al. (2005). De esta manera, estos nuevos conceptos buscan impulsar los compromisos que vayan en armonía con el cuidado con el medio ambiente.

Existen dos normas que comparten una serie de principios comunes pero la aplicación de ambas varía dependiendo del objetivo. En primer lugar, la ISO se compromete a crear normas ambientales internacionales, dando paso a la familia de normas ISO 14.000 creadas con el fin de entregar herramientas a las organizaciones para que puedan medir sus esfuerzos y su actuación en cuanto a asuntos ambientales. Relativa a esta serie de normas es la norma 14.001 la cual 
"establece los criterios para un sistema de gestión ambiental y se puede certificar. Traza un marco que una empresa u organización puede seguir para establecer un sistema de gestión ambiental eficaz". International Organization for Standardization. (2015).

Además, es posible encontrar la serie estandarizada ISO 9000 "describe Ios conceptos y principios fundamentales de la gestión de la calidad que son universalmente aplicables a usuarios específicos." International Organization for Standardization (2015), una de las de las versiones de esta serie es la ISO 9001 que "establece los criterios para un sistema de gestión de la calidad y es el único estándar de la familia que puede certificarse (aunque esto no es un requisito). Puede ser utilizado por cualquier organización, grande o pequeña, independientemente de su campo de actividad." International Organization for Standardization (2021). La diferencia entre ambas series radica en que los sistemas de gestión ambiental no hay un usuario en específico solo requiere el cumplimiento de la normativa y regulaciones, en cambio la ISO 9000 debe asegurar productos de calidad según los requerimientos de usuarios específicos.

A pesar de las diferencias, ambas series normativas persiguen el mismo objetivo, en el que "Ia ISO 14000 (Gestión de la calidad ambiental) aplica, como también lo hace la Norma ISO 9000 (Política de la Calidad), la filosofía del método DEMING: Planificar-Realizar-Controlar-Revisar, como herramienta de mejora continua".( Muriel, 2006, p.3)

Chile no quedó atrás, por lo que adoptó estas normativas traduciendo su versión al español de la Norma Internacional ISO 14001:2015, denominando a su versión NCh-ISO 14.001:2015, al igual que la Norma ISO 9001:2015 en su versión chilena NCh ISO 9001:2015 estas son elaboradas y difundidas por el Instituto Nacional de Normalización (INN).

No obstante, el estado de Chile decide contar con una ley general respecto a materias medio ambientales en el territorio, es por lo que en el año 1994 se promulga la ley 19.300 que propició las Bases Generales del Medio Ambiente. El objetivo de esta ley es "darle un contenido concreto y un desarrollo jurídico adecuado a la garantía constitucional que asegura a todas las personas el derecho a vivir en un medio ambiente libre de contaminación." (Ministerio de Secretaria General de la Presidencia, 2020, p.1). Por lo tanto, se orientan y regulan en base a cuatro principios fundamentales:

1.- El principio preventivo,

2.- El segundo principio es el que contamina paga,

3.- En tercer lugar, el proyecto tiene como principio inspirador el gradualismo, y;

4.- El principio de responsabilidad 
Para la estructura de esta ley se optó por seguir "el modelo de la propuesta de Ley básica de protección ambiental y promoción del desarrollo sostenible de 1993, elaborada para América Latina por el Programa de las Naciones Unidas para el Medio Ambiente (PNUMA)" Bergamini \& Pérez (2015, p.1), Conforme a ello, se da paso a que pueda seguir avanzando en materia medio ambiental con el fin de mejorar la calidad de vida de la sociedad.

Bajo este escenario, este estudio tiene por objetivo evaluar el comportamiento de las siete empresas mineras chilenas, como una contribución a la reflexión sobre la forma de implementación de sistemas de gestión ambiental aporta a la sociedad permitiendo un desarrollo sostenible, más sano para las comunidades, garantizando la continuidad de las

operaciones de las mineras y considerando criterios de impacto ambiental que a nivel internacional toman relevancia para los distintos stakeholders.

Por estas razones, el objetivo general consiste en analizar la implementación de sistemas de gestión ambiental en empresas de la industria minera en Chile. Lo cual se podrá llevar a cabo mediante la descripción del proceso de implementación de Ios SGA en algunas empresas mineras, en conjunto con describir los impactos provocados en el ambiente interno y en los procesos operacionales de las empresas analizadas y explicando sus experiencias y los resultados alcanzados.

El presente artículo sintetiza las temáticas centrales del proyecto de investigación acerca de la implementación de sistemas de gestión ambiental en empresas mineras de Chile. En base a lo anterior el artículo cuenta con el planteamiento del problema y los aspectos principales del marco teórico en la respectiva introducción, posteriormente se señalan la metodología, discusión de resultados y conclusiones.

\section{METODOLOGÍA, MATERIAL Y MÉTODOS}

El enfoque metodológico de la presente investigación es de tipo cualitativa, debido a que la información recopilada busca analizar la implementación de un sistema de gestión ambiental en las empresas, a través de la búsqueda de datos que involucran a la industria de la minería en Chile, con un alcance de sintetización. Con ayuda de la organización y codificación propuesta por el software ATLAS.ti se podrán alcanzar los objetivos propuestos.

El proceso de obtención de información está basado en diversas fuentes tales como artículos de revistas, libros, tesis, páginas web oficiales como ISO.org donde se detallan normativas estándares relacionados al tema de investigación y además se utilizaron informes oficiales de organismos públicos nacionales, bases de datos como Scielo, EBSC0, Dialnet, E-libro y Repositorio CEPAL. Por último, se utilizaron algunos instrumentos detallados en las siguientes tablas. 
El sujeto de esta investigación para la revisión de experiencias se basó en las empresas de la industria minera chilena. De un total de 27 empresas mineras publicadas por la Comisión Chilena del Cobre se escogió una muestra de 7 para la investigación. Las empresas mineras que fueron escogidas como objeto de estudio serán Codelco y ENAMI como empresas estatales y Anglo American Sur, Antofagasta Minerals, BHP Billiton, Compañía Minera del Pacifico y SQM S.A como compañías privadas. Las empresas seleccionadas en la muestra fueron escogidas en base a sus niveles de producción y contribución para el país.

\section{Tabla 1}

Memorias y Reportes de Sustentabilidad 2017- 2020

\begin{tabular}{|c|c|c|}
\hline \multicolumn{3}{|c|}{ Instrumentos para la recolección de información } \\
\hline \multirow[t]{4}{*}{ MEMORIAS } & CODELCO & $\begin{array}{l}\text { - Memoria anual } 2020 \\
\text { - https://n9.cl/7wkir } \\
\text { - Memoria anual } 2019 \\
\text { - https://n9.cl/sn8th } \\
\text { - Memoria anual 2018 } \\
\text { - https://n9.cl/7bg6j } \\
\text { - Memoria anual 2017 } \\
\text { - https://n9.cl/dmzb9 }\end{array}$ \\
\hline & Anglo Sur & $\begin{array}{l}\text { Memorias disponibles para descarga en página } \\
\text { de la Comisión del Mercado Financiero: } \\
\text { https://n9.cl/1glce } \\
\text { - Memoria anual } 2020 \\
\text { - Memoria anual } 2019 \\
\text { - Memoria anual } 2018 \\
\text { - Memoria anual } 2017\end{array}$ \\
\hline & ENAMI & $\begin{array}{l}\text { - Memoria anual } 2020 \\
\text { - https://n9.cl/a91ap } \\
\text { - Memoria anual } 2019 \\
\text { - https://n9.cl/w1z7d } \\
\text { - Memoria anual } 2018 \\
\text { - https://n9.cl/hse4r } \\
\end{array}$ \\
\hline & Grupo CAP & $\begin{array}{l}\text { - Memoria anual } 2020 \\
\text { - } \text { https://n9.cl/svzcd } \\
\text { - } \text { Memoria anual } 2019 \\
\text { - https://n9.cl/12qks } \\
\text { - Memoria anual } 2018 \\
\text { - https://n9.cl/t50gr } \\
\text { - Memoria anual } 2017 \\
\text { - } \text { https://n9.cl/pel7 }\end{array}$ \\
\hline
\end{tabular}




\begin{tabular}{|c|c|c|}
\hline & Soquimich S.A. & $\begin{array}{l}\text { - Memoria anual } 2020 \\
\text { - https://n9.cl/w3hnx } \\
\text { - Memoria anual } 2019 \\
\text { - https://n9.cl/hclym } \\
\text { - Memoria anual } 2018 \\
\text { - https://n9.cl/tb8yx } \\
\text { - Memoria anual 2017 } \\
\text { - https://n9.cl/p7x9a }\end{array}$ \\
\hline & $\begin{array}{l}\text { Antofagasta } \\
\text { Minerals }\end{array}$ & $\begin{array}{l}\text { - Informe anual y estados financieros } 2020 \\
\text { - https://n9.cl/1ud36 } \\
\text { - Informe anual y estados financieros } 2019 \\
\text { - https://n9.cl/fryh } \\
\text { - Informe anual } 2018 \\
\text { - https://n9.cl/72vbq } \\
\text { - Informe anual } 2017 \\
\text { - https://n9.cl/luih }\end{array}$ \\
\hline & BHP Billiton & $\begin{array}{l}\text { 1.-Minera Cerro Colorado: Disponibles para } \\
\text { descarga en página de la Comisión del Mercado } \\
\text { Financiero: } \\
\text { https://n9.cl/ft3ae } \\
\text { - Memoria anual } 2020 \\
\text { - Memoria anual } 2019 \\
\text { - Memoria anual } 2018 \\
\text { - Memoria anual } 2017 \\
\text { 2.- Minera Spence: Disponibles para descarga en } \\
\text { página de la Comisión del Mercado Financiero: } \\
\text { https://n9.cl/7ij3ug } \\
\text { - Memoria anual } 2020 \\
\text { - Memoria anual } 2019 \\
\text { - Memoria anual } 2018 \\
\text { - Memoria anual } 2017\end{array}$ \\
\hline $\begin{array}{l}\text { REPORTES DE } \\
\text { SUSTENTABILIDAD }\end{array}$ & CODELCO & $\begin{array}{l}\text { - Reportes de sustentabilidad } 2020 \\
\text { - https://n9.cl/xjhqv } \\
\text { - } \text { Reportes de sustentabilidad } 2019 \\
\text { - https://n9.cl/jnm78w } \\
\text { - Reportes de sustentabilidad } 2018 \\
\text { - https://n9.cl/60ivi } \\
\text { - Reportes de sustentabilidad } 2017 \\
\text { - https://n9.cl/mjfw }\end{array}$ \\
\hline
\end{tabular}




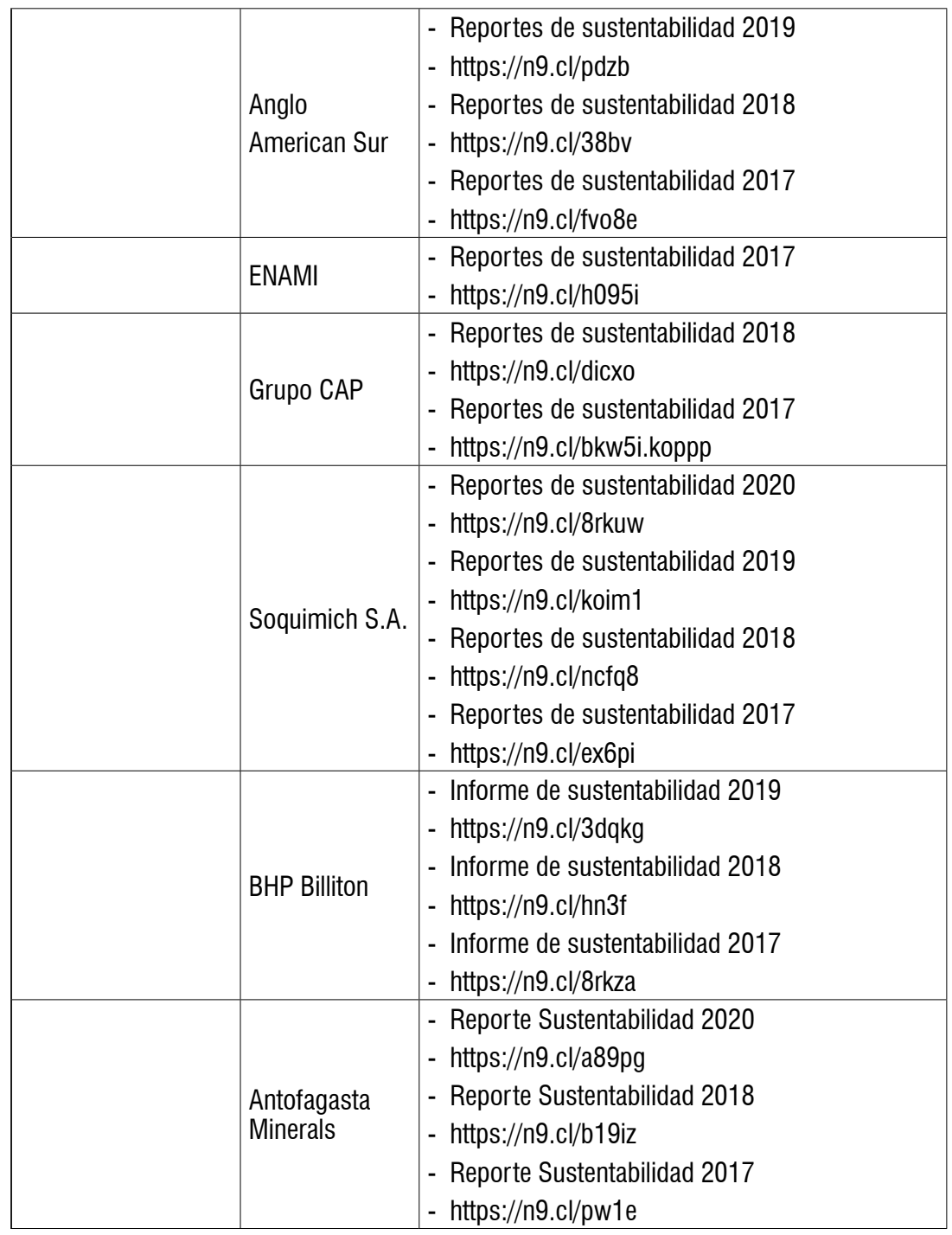

Fuente: Elaboración propia

Los criterios de calidad para ser usados en el análisis y evaluación de la investigación fueron:

1. Credibilidad: La información y datos han sido recopilados a través de fuentes fidedignas, el análisis documental se da por medio de la revisión de documentación presentada por organismos públicos en base a datos autorizados.

2. Transferibilidad: Bajo el contexto de la investigación, la gestión ambiental puede ser transferida a otro contexto similar preservando 
los significados e interpretaciones y lograr un entendimiento más profundo.

3. Confirmabilidad: Por medio de las afirmaciones de distintos autores que fueron investigados, se pudo llegar a hallazgos similares que permitieron llevar a cabo el análisis.

\section{RESULTADOS}

Las empresas seleccionadas para el análisis corresponden a las más grandes empresas mineras en Chile, y considerando su contribución a la economía del país y a la participación en el mercado internacional, son objeto de observación para políticos, parlamentarios, organizaciones sociales y medios de comunicación.

La presión de todos estos agentes con intereses contrapuestos deja en efecto que las empresas en cuestión revelan gran cantidad de información en sus memorias y reportes de sustentabilidad, en las que muestran que cumplen con estándares medioambientales y de que mantienen la mayor eficiencia posible en materias medioambientales, esto incluyendo integración de nuevas tecnologías y un trabajo profundo en la estructuración de sus valores y cultura organizacional. Sin embargo, se evidencia en sus documentos poca información de las inversiones y la manera en que logran resultados que son destacados en sus reportes.

Un sistema de gestión ambiental es lo que hoy en día garantiza la continuidad de las operaciones de las empresas mineras, las cuales se esfuerzan por integrar sus actividades hacia una mejora continua que las acerque a un desarrollo más sostenible como lo mencionan Robbins \& Coulter (2005). Integrar un sistema de gestión en la empresa y convertirlo en un valor fundamental de la misma, significa según Van Hoof et al. (2008), acercarse a alcanzar un equilibrio entre los problemas de contaminación y el desarrollo económico. Las empresas se especializan en investigación e invierten en mejorar sus gestiones, cada organización según sus necesidades y capacidades optan por modelos de sistemas de gestión ambiental que guían y dan pauta a las acciones a seguir.

Es relevante como además del ámbito internacional, las comunidades locales exigen sus derechos, ocasionando que la industria minera se muestre más comprometida, evolucionando en su relación, fortaleciendo la responsabilidad social por medio de la inversión en el desarrollo social, cultural y ambiental de las comunidades.

El escenario actual se visualiza como una oportunidad para las empresas, las cuales prometen ser parte de una industria 4.0, que busca apegarse a las políticas que benefician al desarrollo sustentable y que puede ser la única forma de su subsistencia en el futuro. 


\section{DISCUSIÓN Y CONCLUSIÓN}

Chile, un país rico en recursos naturales que ha sido sujeto de explotación a lo largo del tiempo, dónde sin duda ha estado muy presente la industria minera, la cual desde el siglo XX ha ido posicionando al país como un productor importante de minerales a nivel mundial, antiguamente con una cantidad menor la cual ha crecido de forma exagerada en las últimas décadas.

La implementación de los sistemas de gestión ambiental ha sido fundamental para la industria minera, entregando criterios necesarios para cumplir con los objetivos del desarrollo sostenible propuesto por la Organización de las Naciones Unidas. Se ha trabajado en ello por medio de la generación de lazos con los grupos de interés demostrando el compromiso y una reputación de responsabilidad social a nivel nacional e internacional.

Cabe destacar que han logrado incluir la sustentabilidad como un valor fundamental en sus operaciones, integrando tecnologías innovadoras para conseguir la eficiencia energética y sometiéndose continuamente a evaluaciones que incentivan seguir fortaleciendo sus sistemas de control y gestión.

Para poder llevar a cabo la ejecución de los planes mineros sustentables, es necesario definir los compromisos, que deben ser medidos dentro de un plazo, sumando la concreción de los proyectos que mitiguen los efectos ambientales negativos, internalizar la minería responsable con tecnología, y la evaluación de los resultados obtenidos en términos cualitativos y cuantitativos.

Se logra observar que se genera una situación similar en cada minera: una promesa contundente, con altas expectativas de manejo medioambiental y compromisos de reducción de contaminación a corto y largo plazo. No obstante, las memorias y reportes de sustentabilidad consultados demuestran una falta de información, respecto a las inversiones realizadas en proyectos de mejoramiento ambiental y al manejo de cifras cuantitativas que evidencien el real avance que han generado. Aquello puede llevar a cuestionamientos del usuario, dando a conocer que no hay claridad en estos aspectos y quedando al debe. Por ende, es necesario que se tome como una oportunidad de mejora para todas las mineras.

Por otra parte, se llevó a cabo el análisis de datos realizados con el software Atlas Ti que permitió destacar algunos códigos que tenían mayor relación de frecuencia entre sí, logrando concluir en base a ellos.

Principalmente se encontraron altas relaciones entre las subcategorías de sustentabilidad y estrategia ambiental, aquello se debe a que cada una de las empresas tiene por objetivo ser la minera del futuro, reduciendo los costos de producción y los riesgos medioambientales, con planes tecnológicos y operacionales, aquellos solo se puede llevar a cabo, por medio de una estrategia donde su valor central sea la sustentabilidad, para lograr adaptarse a las necesidades de la comunidad y del planeta. 
Por su parte, el resultado entre medio ambiente y desarrollo sostenible sugiere un reconocimiento a los compromisos que se han propuesto las mineras, el trabajo en materias de protección ambiental e inclusión social se ven reflejados de manera integral.

Por último, se ha identificado una vinculación entre sistema de gestión y estrategia ambientales, ambos conceptos trabajan en conjunto para incrementar la eficiencia y la competitividad en el mercado, y se concreta mediante la creación de equipos de gerencias y líneas de direccionamiento estratégico que potencian el trabajo en esas áreas.

Las nuevas estrategias implican darle a la sustentabilidad un pilar fundamental y que sea capaz de crear valor a las empresas, donde la dirección debe ser muy activa para integrar los valores y otorgar una importante ventaja competitiva. Después de todo, se está hablando del futuro y no tan solo el de la industria

\section{REFERENCIAS BIBLIOGRÁFICAS}

ACUÑA, N., FIGUEROA, L., \& WILCHES, M. (2017). Influencia de los Sistemas de Gestión Ambiental ISO 14001 en las organizaciones: caso estudio empresas manufactureras de Barranquilla. Ingeniare. Revista chilena de ingeniería. 25(1), 143-153 http://dx.doi.org/10.4067/S071833052017000100143

ALAÑA, T., MORÁN, G., \& SANMARTíN, G. (2017). La auditoría ambiental en las MIPYMES como herramienta de control interno en la gestión empresarial. , 9(1), 143-147. Recuperado a partir de https://rus.ucf.edu. cu/index.php/rus/article/view/527

BECERRA, F., ANDRADE, A., \& DÍAZ, L. (2019). Sistema de gestión de la calidad para el proceso de investigación: Universidad de Otavalo, Ecuador. Actualidades Investigativas en Educación. 19(1), 571-604. Doi: http:// dx.doi.org/10.15517/aie.v19i1.35235

BERGAMINI, K., \& PÉREZ, C. (2015). Fiscalización y cumplimiento ambiental en Chile: principales avances, desafíos y tareas pendientes. EURE (Santiago). 41(124), 267-277. Doi: http://dx.doi.org/10.4067/S025071612015000400013

COGLIANESE, C., \& NASH, J. (2001). Fortalecimiento de la gestión ambiental del sector privado. Issues in science and technology. 17(3) Recuperado de https://issues.org/coglianese/

ESQUIVEL, A., ROBAINA, R., \& CASTELLANOS, G. (2017). Mejora continua de los procesos de gestión del conocimiento en instituciones de educación superior ecuatorianas. Retos de Dirección. 11(2), 56-72 Recuperado 
el 13 de Junio de 2021, de http://scielo.sld.cu/scielo.php?script=sci_ abstract\&pid=S2306-91552017000200005\&Ing =es\&nrm =iso

FERRARI, G. (2012). Impacto ambiental de las actividades económicas. Septem Ediciones. Recuperado el 15 de Junio de 2021, de https://elibro. net/es/ereader/uvalparaiso/42040?page $=62$

FILIPPONE, J., CANDELA, A., LÓPEZ, A., \& ORIHUELA, R. (2005). Diseño Ecoeficiente de Envases y Embalajes No Reutilizables. Información tecnológica. 16(3), 57-61.Doi: http://dx.doi.org/10.4067/S071807642005000300008

GALLOPÍN, G. (2003). Sostenibilidad y desarrollo sostenible: un enfoque sistémico. CEPAL. Recuperado el 26 de mayo de 2021, de http://hdl. handle.net/11362/5763

GIDDENS, A. (1999). Un mundo desbocado. Taurus. Recuperado el 27 de mayo de 2021, de https://www.academia.edu/43342234/_UN_MUND0_ DESBOCADO_DE_ANTHONY_GIDDENS

INTERNATIONAL ORGANIZATION FOR STANDARDIZATION. (2015). Norma ISO 14.001. International Organization for Standardization. Recuperado el 10 de junio de 2021, de https://www.iso.org/obp/ui\#iso:std:iso:14001:ed3:v1:es

INTERNATIONAL ORGANIZATION FOR STANDARDIZATION. (2021). ISO. Recuperado el 25 de mayo de 2021, de ISO: https://www.iso.org/aboutus.html

INTERNATIONAL ORGANIZATION FOR STANDARDIZATION. (2021). ISO org. Recuperado el 26 de mayo de 2021, de https://www.iso.org/iso-14001environmental-management.html

INTERNATIONAL ORGANIZATION FOR STANDARDIZATION. (S.F.). ISO. Recuperado el 25 de mayo de 2021, de https://www.iso.org/ standard/45481.html

ISO, E. (2015). ISO 14001: Los requisitos del Sistema de Gestión Ambiental. Recuperado de ISO Tools: https://www.nueva-iso-14001.com/quienessomos/

KAMMERBAUER, J. (2001). Las dimensiones de la sostenibilidad: Fundamentos ecológicos, modelos paradigmáticos y senderos. Inter ciencia. 26(8), 353-359. Recuperado el 06 de junio de 2021, de http://ve.scielo.org/ scielo.php?script=sci_arttext\&pid=S0378-18442001000800006

LUDEVID, M. (2004). La gestión ambiental de la empresa. Editorial Ariel S.A https://elibro.net/es/ereader/uvalparaiso/48251?page $=44$ 
MINISTERIO SECRETARÍA GENERAL DE LA PRESIDENCIA. (2020). Biblioteca Nacional de Chile; Ley Chile. Recuperado el 16 de junio de 2021, de https://www.bcn.cl/leychile/navegar?idNorma $=30667$

MORENO, I., \& ROSERO, L. (2000). DSpace de ESPOL. Recuperado el 05 de junio de 2021, de http://www.dspace.espol.edu.ec/xmlui/ handle/123456789/3563

MULDER, K. (2007). Desarrollo sostenible para ingenieros. UPC. Recuperado el 24 de mayo de 2021, de https://elibro.net/es/ereader/ uvalparaiso/61440? page $=15$

MURIEL, R. (2006). Gestión ambiental. Ideas sostenibles. Recuperado el 17 de junio de 2021, de https://upcommons.upc.edu/bitstream/ handle/2099/1110/13_GestAmbientalRafaelMuriel_cast. pdf? sequence $=1$ \&isAllowed $=y$

PALOMO, A. (2012). Apuntes teóricos para el estudio de la Globalización desde la perspectiva de las Relaciones Internacionales. Confines de relaciones internacionales y ciencia política, 8(16), 69-109 Recuperado el 25 de mayo de 2021, de http://www.scielo.org.mx/scielo.php?script=sciabstr act\&pid $=$ S187035692012000200004\&lng $=$ es\&nrm $=$ iso

PEREA, S., CABRALES, J., \& GIMÉNEZ, H. (2009). Sostenibilidad y Desarrollo. Alarife, Revista de Arquitectura. Recuperado el 02 de junio de 2021, de https://dialnet.unirioja.es/servlet/articulo?codigo $=3195185$

PÉREZ, R. (2019). Los objetivos de desarrollo sostenible. Librería Bosch. Recuperado el 27 de mayo de 2021, de https://elibro.net/es/ereader/ uvalparaiso/130477? page $=19$

THOMPSON, A., JOHN, G., PETERAF, M., \& STRICKLAND, A. (2018). Administración estratégica. Recuperado el 27 de Mayo de 2021, de https://www.academia.edu/28414430/Administraci\%C3\%B3n_ Estrat\%C3\%A9gica_Thompson_18va

PORTER, M. E. (2014). Estrategia competitiva: técnicas para el análisis de los sectores industriales y de la competencia (2a. ed.). Grupo Editorial Patria. https://elibro.net/es/lc/uvalparaiso/titulos/114079

PRIETO, M. (2011). Sistemas de gestión ambiental. España: AENOR (Asociación Española de Normalización y Certificación). Recuperado el 14 de junio de 2021, de https://elibro.net/es/ereader/uvalparaiso/53563?page $=13$

ROBBINS, S. P. COULTER, M. Y PINEDA AYALA, L. E. (TRAD.). (2018). Administración (13a. ed.). Pearson Educación. https://elibro.net/es/lc/ uvalparaiso/titulos/113242 
SUMBA, Y., \& LOURDES, K. (2018). Las microempresas y la necesidad de fortalecimiento: reflexiones de la zona sur de Manabí, Ecuador. Revista Universidad y Sociedad, 10(5), 323-326 Recuperado el 27 de junio, 2021, desde http://scielo.sld.cu/scielo.php?script=sci_ arttext\&pid=S2218-36202018000500323

VAN HOOF, B., MONROY, N., \& SAER, A. (2008). Producción más limpia: paradigma de gestión ambiental. Colombia: Universidad de los Andes. Recuperado el 07 de junio de 2021, de https://elibro.net/es/ereader/ uvalparaiso/40312 